23

\title{
Аналитическое описание спектральных характеристик сенсора коэффициента преломления на основе отражательного интерферометра
}

\author{
(C) B.C. Терентьев, В.А. Симонов \\ Институт автоматики и электрометрии Сибирского отделения РАН, \\ 630090 Новосибирск, Россия \\ e-mail: terentyev@iae.nsk.su
}

Поступила в редакцию 19.02.2021 г.

В окончательной редакции 01.04.2021 г.

Принята к публикации 05.04.2021 г.

\begin{abstract}
Впервые представлены аналитические формулы, описывающие спектральные характеристики сенсора на основе отражательного интерферометра для наклонного падения света в оптической схеме Кречмана. Данный сенсор предназначен для измерения коэффициента преломления аналитической среды (жидкости, газа) за счет эффекта полного внутреннего отражения в структуре на основе металло-диэлектрического многослойного покрытия. Определены параметры тонкой металлической пленки, входящей в состав структуры, необходимые для оптимизации спектрального профиля сенсора, который представляет собой спектральные максимумы с варьируемой шириной. В качестве диэлектрического покрытия использованы четвертьволновые слои для наклонного падения света. Приведены аналитические зависимости характеристик сенсора от поляризации: разрешающая способность, чувствительность и контраст, а также параметр качества. Теоретически параметр качества сенсора может иметь бесконечно высокие значения для обеих поляризаций, однако практически он ограничивается потерями в слоях структуры. Показано, что выгоднее использовать $S$-поляризацию за счет большей добротности резонатора в структуре сенсора при том же числе диэлектрических слоев. Предложен метод изготовления структуры сенсора с оптическим контролем в процессе вакуумного напыления.
\end{abstract}

Ключевые слова: отражательный интерферометр, полное внутреннее отражение, сенсор показателя преломления, инвертированный поверхностный плазмонный резонанс.

DOI: $10.21883 /$ OS.2021.08.51207.1932-21

\section{1. Введение}

Сенсоры на основе эффекта нарушенного полного внутреннего отражения применяются для измерения коэффициента преломления газов, жидкостей, в частности, в биологических приложениях $[1,2]$, поэтому их исследование активно ведется в настоящее время. Вариантами реализации таких сенсоров являются разные оптические схемы, в частности схема Кречмана на основе призмы, или иные схемы на основе дифракционной структуры, в которых в качестве сигнального репера используется узкий спектральный минимум интенсивности в отражении, который в литературе имеет устоявшееся название поверхностного плазмонного резонанса (ППР, surface plasmon resonance, SPR) и который является результатом максимизации поглощения света в сенсорной структуре $[3,4]$. В настоящей работе исследованы свойства одного из новых вариантов сенсоров, использующих эффект полного внутреннего отражения и поверхностного плазмонного резонанса в конфигурации Кречмана. Сенсор имеет „инвертированный“ спектральный профиль в отраженном свете, подобный исследованному в [5]. Сигнальным репером в такой схеме служит узкий спектральный максимум интенсивности, который является результатом минимизации поглощения света в сенсорной структуре, в англоязычной литературе такой тип резонанса называется инвертированным поверхностным плазмонным резонансом (ИППР, inverted surface plasmon resonance - ISPR) [6]. Исследуемый тип сенсора может иметь очень высокие параметры качества, превышающие 1000 за счет многолучевой интерференции в базовом слое, а также имеет потенциальную возможность значительно увеличить параметр качества за счет сужения спектральной ширины сигнального максимума в лазерной схеме при создании положительной обратной связи, что принципиально невозможно для ППР-сенсоров. Поэтому разработка различных схем и методик реализации ИППР-сенсоров актуальна и имеет практический интерес. Однако для этого необходимо осветить некоторые моменты, связанные с оптимизацией характеристик подобных сенсоров, структуры металлодиэлектрических слоев [7,8].

Основная цель данной работы состоит в получении аналитических формул, описывающих оптические свойства металло-диэлектрического покрытия ИППРсенсора. Для этого используются приближенная модель проводящей поверхности, матричный метод и теория отражательного интерферометра (ОИ). На основе данных методов можно определить оптимальные парамет- 


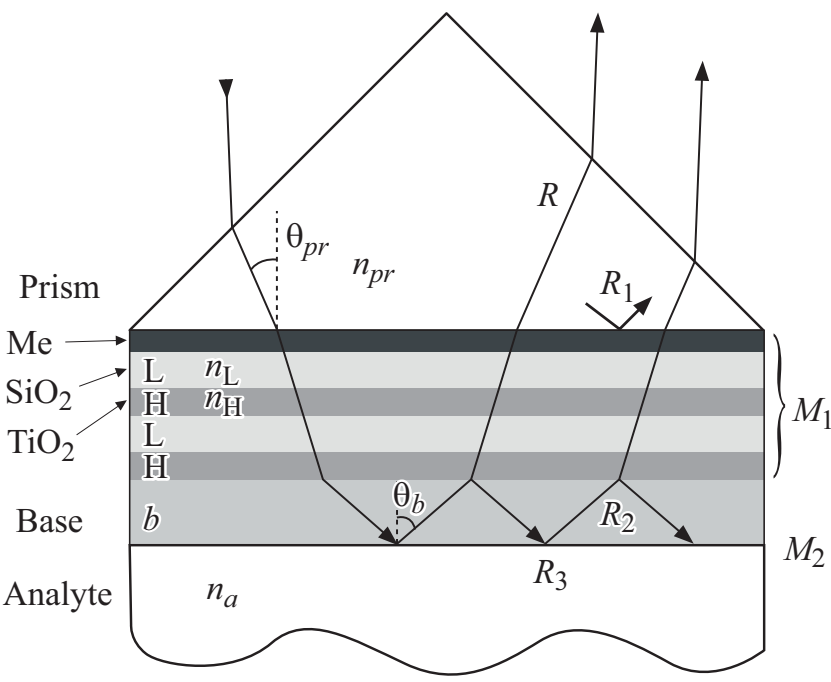

Рис. 1. Оптическая схема сенсора на основе наклонного ОИ: Prism - призма $\left(n_{p r}\right)$, Ме - тонкая металлическая пленка, диэлектрические слои из материала с высоким $\left(H, n_{H}\right)$ и низким $\left(L, n_{L}\right)$ коэффициентами преломления, $b$, Base базовый слой $\left(n_{b}\right), a$, Analyte - аналит $\left(n_{a}\right), \theta_{p r}-$ угол падения в призме, $\theta_{b}-$ угол падения в базовом слое, $R_{1,2}-$ коэффициенты отражения зеркала $M_{1}, R_{3}$ - зеркала $M_{2}$.

ры многослойного покрытия, металлической пленки и основные характеристики сенсора: разрешающую способность (ширину на полувысоте спектрального пика $\delta \lambda)$, спектральную чувствительность $S_{\lambda}$, контраст $C_{R}$ и порядок качества $F O M$, а также предложить экспериментальную методику изготовления сенсора.

\section{2. Теория ОИ для наклонного падения света}

Структура сенсора представлена на рис. 1. На грань призмы нанесена тонкая металлическая пленка $(\mathrm{Me})$ и диэлектрическое многослойное покрытие. Целесообразно многослойное покрытие рассматривать раздельно, как переднее зеркало $M_{1}$, базу и заднее зеркало $M_{2}$. Предполагается получить аналитические формулы для коэффициентов зеркала $M_{1}$, а затем использовать их совместно с формулой, полученной из теории ОИ для определения спектральных характеристик сенсора.

В работе [5] показано, что при использовании нечетвертьволновых диэлектрических слоев, к тому же неодинаковой оптической толщины, специальным образом согласованных с тонкой металлической пленкой, можно получить спектральный профиль отражения $R(\lambda)$ с узкими максимумами шириной на полувысоте $\delta \lambda$. Такой профиль подобен профилю интерферометра Фабри-Перо для нормального падения света в пропускании. Данная система характеризуется практически идеальным контрастом $C_{R}$, близким к 1 , что делает максимально возможным параметр качества FOM (Figure of Merit), который можно определить формулой:

$$
F O M=C_{R} \frac{S_{\lambda}}{\delta \lambda}=C_{R} \frac{\Delta \lambda}{\pi \delta \lambda} \frac{d \varphi}{d n_{a}} .
$$

Здесь $\Delta \lambda$ - область свободной дисперсии, определяемая базой ОИ, $\varphi-$ набег фаз между зеркалами $M_{1}$ и $M_{2}$, $n_{a}$ - коэффициент преломления аналитической среды (газа, жидкости и т.п.), $S_{\lambda}$ - спектральная чувствительность к изменению $n_{a}$. Однако такую систему аналитически достаточно сложно описать из-за нелинейной зависимости оптической толщины каждого диэлектрического слоя от его номера. Кроме этого, с точки зрения разработки методов практического изготовления структуры ИППР-сенсора может быть преимуществом наносить одинаковые по оптической толщине (для своего коэффициента преломления) слои вместо неодинаковых. Поэтому в данной работе рассматривается структура со слоями одинаковой оптической толщины, которая при определенном выборе материала металлической пленки может иметь контраст $C_{R} \geq 0.9$, лишь незначительно снижающий максимальное значение FOM.

Если известны $\Psi_{1,2}, \Phi_{1}$ - фазы коэффициентов отражения и пропускания соответственно $R_{1,2}$ и пропускания $T_{1}=T_{2}$ зеркала $M_{1}, \Psi_{3}$ - фаза и коэффициент отражения $R_{3}$ заднего зеркала $M_{2}$, то можно записать формулу аппаратной функции ОИ $[9,10]$ :

$$
\begin{gathered}
R(\varphi)=R_{1}+2 T_{1} \sqrt{R_{1} R_{3}} \frac{\cos (\vartheta+2 \varphi)-\sqrt{R_{2} R_{3}} \cos (\vartheta)}{1+R_{2} R_{3}-2 \cos (2 \varphi) \sqrt{R_{2} R_{3}}} \\
+\frac{R_{3} T_{1}^{2}}{1+R_{2} R_{3}-2 \cos (2 \varphi) \sqrt{R_{2} R_{3}}} \\
\vartheta=\Psi_{1}+\Psi_{2}-2 \Phi_{1} \\
\varphi\left(\theta_{b}, \lambda\right)=\frac{2 \pi n_{b} h_{b} \cos \theta_{b}}{\lambda}-\frac{\Psi_{2}\left(\theta_{b}, \lambda\right)+\Psi_{3}\left(\theta_{b}, \lambda\right)}{2}
\end{gathered}
$$

где $\lambda$ - длина волны, $\theta_{b}$ - угол падения света на зеркала внутри резонатора, т. е. в слое базы, $h_{b}-$ физическая толщина, $n_{b}$ - показатель преломления базы ОИ, $R(\varphi)$ справедливо для $S$ - и $P$-поляризаций с подстановкой соответствующих коэффициентов $R_{1,2,3}, T_{1}$ и их фаз для этих поляризаций. В условиях полного внутреннего отражения на границе базы и внешней среды $R_{3}=1$, и этот параметр можно было бы удалить из формулы (2), оставив зависимость от параметров зеркала $M_{2}$ только через фазу $\Psi_{3}(\theta, \lambda)$. Однако в более общем случае присутствие потерь света на паразитное поглощение или рассеяние либо в слое базы, либо на границе раздела $M_{2}$ эффективно приводит к снижению $R_{3}$ и влияет на $R$. Как видно из (2), коэффициент отражения представляет собой сложную функцию, состоящую из трех членов. Первые два члена в (2) стремятся к нулю, если $R_{1} \rightarrow 0$, в этом случае зависимость $R(\varphi)$ с точностью до константы будет подобна пропусканию интерферометра ФабриПеро (ИФП). Если же $R_{1} \neq 0$ и $\vartheta \neq(2 m+1) \pi(m-$ целое), что характерно для систем с потерями, то форма 

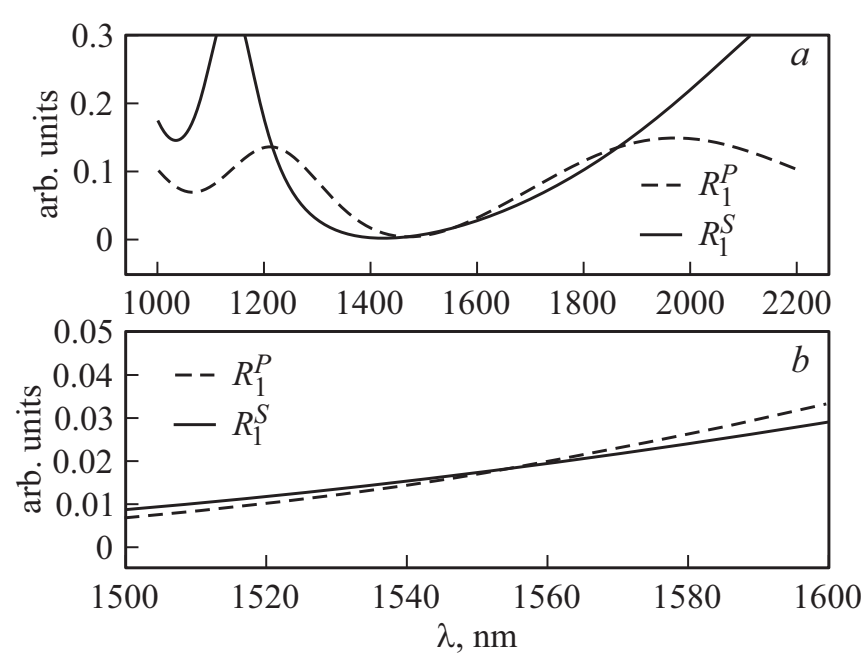

Рис. 2. Расчетные спектры коэффициентов отражения $R_{1}^{[S, P]}$ для $N=3$ и двух поляризаций в спектральном диапазоне: (a) $1000-2200 \mathrm{~nm},($ b) $1500-1600 \mathrm{~nm}$.

профиля $R(\varphi)$ будет асимметрична (в пропускании ИФП форма профиля симметрична).

Таким образом, ключевым элементом для получения ИППР интерференционной картины в отраженном свете в виде узких максимумов интенсивности является асимметричное по коэффициентам отражения переднее зеркало $M_{1}$ (рис. 1). Его коэффициенты отражения, пропускания и поглощения и их оптимизацию можно определить с помощью матричных формул [11].

\section{3. Аналитические формулы для коэффициентов переднего зеркала}

Данные аналитические формулы непосредственно раскрывают смысл использования тонкой металлической пленки, ее роль в формировании асимметрии коэффициентов отражения зеркала $M_{1}$ и интерференционной картины в отражении от наклонного ОИ в условиях ПВО. $\mathrm{C}$ их помощью можно определить, какие характеристики пленки металла необходимы, чтобы получать оптимальные параметры переднего зеркала $\left(R_{1} \rightarrow 0, R_{2} \rightarrow 1\right)$. Как известно, для нахождения коэффициентов отражения $r_{j}$ и пропускания $t_{j}$ произвольного слоя с коэффициентом преломления $n_{j}$ и толщиной $h_{j}$, расположенного между двумя полупространствами диэлектриков с коэффициентами преломления $n_{p r}$ (полупространство падения и отражения света, призма) и $n_{a}$ (полупространство пропускания), можно использовать элементы характеристической матрицы среды, записанной в следующем виде:

$$
Z=\left|\begin{array}{cc}
\cos \alpha_{j} & \frac{i s \sin \alpha_{j}}{u_{j}} \\
i u_{j} \sin \alpha_{j} & \cos \alpha_{j}
\end{array}\right|
$$

где $\alpha_{j}=h_{j} k n_{j} \cos \theta_{j}, k=2 \pi / \lambda, \theta_{j}-$ угол падения света в слое, который определяется из закона преломления, причем $\cos \theta_{j}=\sqrt{1-\left(n_{p r} \sin \theta_{p r} / n_{j}\right)^{2}}, \quad \operatorname{Im}\left(\cos \theta_{j}\right) \leq 0$, где $\theta_{p r}$ - угол падения света в призме. Так как падение света предполагается наклонным, матрица $Z$ записывается для $S$ - и $P$-поляризации через коэффициенты:

$$
u_{j}= \begin{cases}u_{j}^{[S]}=n_{j} \cos \theta_{j} & (S-\text { поляризация }) \\ u_{j}^{[P]}=n_{j} \cos \theta_{j} & (P-\text { поляризация })\end{cases}
$$

Матрица $Z$ справедлива и для комплексного коэффициента преломления. Тогда коэффициенты отражения и пропускания находятся по формулам:

$$
\begin{aligned}
& r_{j}=\frac{u_{p r}\left(Z_{11}+Z_{12} u_{a}\right)-\left(Z_{21}+Z_{22} u_{a}\right)}{u_{p r}\left(Z_{11}+Z_{12} u_{a}\right)+\left(Z_{21}+Z_{22} u_{a}\right)}, \\
& t_{j}=\frac{2 \sqrt{u_{p r} u_{a}}}{u_{p r}\left(Z_{11}+Z_{12} u_{a}\right)+\left(Z_{21}+Z_{22} u_{a}\right)},
\end{aligned}
$$

где $Z_{i j}$ - элементы матрицы, $i, j=1,2$. Энергетические коэффициенты можно найти по формулам $R_{j}=\left|r_{j}\right|^{2}, T_{j}=\left|t_{j}\right|^{2}$. Для любой системы слоев можно записать характеристическую матрицу как последовательное их произведение, начиная от первого слоя, на который падает свет. В нашем случае первым слоем является металлический слой с параметрами $h_{m}$ и $\hat{n}_{m}=n_{m}-i \chi_{m}$, где действительные числа $n_{m}, \chi_{m}>0$ - коэффициенты преломления и поглощения слоя соответственно. Когда толщина слоя $h_{m} \leq \lambda / 20$, можно сделать разложение матричных элементов (4) до первого порядка малости с подстановкой $\cos ^{2} \theta_{m}=1-\left(n_{p r} \sin \theta_{p r} / \hat{n}_{m}\right)^{2}$ :

$$
\begin{aligned}
& Z_{m}^{[S]} \approx\left[\begin{array}{cc}
1 & i h_{m} k \\
i h_{m} k\left(\hat{n}_{m}^{2}-n_{p r}^{2} \sin ^{2} \theta_{p r}\right) & 1
\end{array}\right] \\
& \approx\left[\begin{array}{cc}
1 & 0 \\
\xi^{[S]} & 1
\end{array}\right],
\end{aligned}
$$

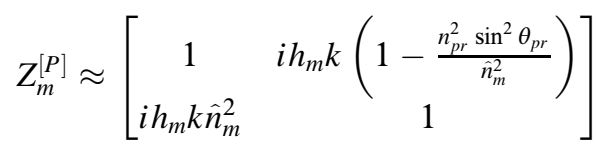

$$
\begin{aligned}
& \approx\left[\begin{array}{cc}
1 & 0 \\
\xi^{[P]} & 1
\end{array}\right],
\end{aligned}
$$

где поверхностные проводимости:

$$
\begin{aligned}
& \xi^{[S]}= \xi_{1}^{[S]}+i \xi_{2}^{[S]}=2 k h_{m} n_{m} \chi_{m} \\
&+i k h\left(\left(n_{m}^{2}-\chi_{m}^{2}\right)-n_{p r}^{2} \sin ^{2}\left(\theta_{p r}\right)\right), \\
& \xi^{[P]}=\xi_{1}^{[P]}+i \xi_{2}^{[P]}=2 k h_{m} n_{m} \chi_{m}+i k h\left(n_{m}^{2}-\chi_{m}^{2}\right),
\end{aligned}
$$

определены через параметры металлической пленки. Примечательно, что в случае $S$-поляризации оказывают влияние и параметры среды падения света, т. е. $\xi_{2}^{[S]} \neq \xi_{2}^{[P]}$. Из (8) следует, что $\xi_{1}^{[P, S]}>0$, и для большинства металлов $\xi_{2}^{[P, S]}<0$. Но последнее неравенство справедливо только для коэффициентов массивных металлов. 
В случае тонкой пленки $(<20 \mathrm{~nm})$ ее свойства могут заметно отличаться от массивного металла и реальные $n_{m} \chi_{m}$ будут иметь другие значения и соотношения изза образования гранулированного покрытия [12]. Правый верхний элемент в матрицах (7) можно приравнять к нулю, так как в случае металлов практически всегда выполняется неравенство $\left|\hat{n}_{m}^{2}\right| \gg 1$. Такое приближение называется приближением или методом проводящей поверхности (МПП) [9], оно достаточно точно (около $1 \%$ величины амплитудных коэффициентов) и позволяет получать простые и понятные аналитические формулы.

Для выражения коэффициента отражения $R_{1}^{[S, P]}$ зеркала $M_{1}$ можно рассмотреть структуру, состоящую из четного числа слоев диэлектрика, последовательно чередующихся с низким $n_{L}$ и высоким $n_{H}$ показателями преломления, расположенных на металлической пленке, начиная со слоя с $n_{L}$. Характеристическая матрица $Z_{L H, N}$ набора из $N$ таких сдвоенных слоев для падения света из среды с коэффициентом преломления $n_{p r}$ и выходной средой с коэффициентом преломления $n_{b}$ (база) описывается выражениями, включающими полиномы Чебышёва второго рода степеней $N-1$ и $N-2$, если толщины слоев в двуслойнике произвольны [11]. В этом случае матричные элементы характеристической матрицы имеют сложный аналитический вид. Однако их можно значительно упростить, если предположить, что многослойное покрытие представляет собой набор четвертьволновых слоев, в которых при наклонном падении света происходит набег фаз $\pi / 2$, т.е. толщина слоев равна $h_{j}=\pi /\left(2 k_{0} n_{j} \cos \theta_{j}\right), j=L, H, k_{0}=2 \pi / \lambda_{0}, \lambda_{0}-$ центральная длина волны, $n_{L}<n_{H}$. Это обусловлено созданием в резонаторе интерферометра, т.е. в его базе, как можно большего числа эффективно интерферирующих лучей за счет максимизации коэффициента отражения $R_{2}^{[S, P]}$, что и происходит при использовании четвертьволновых покрытий. Также желательно минимизировать зависимость $R_{2}^{[S, P]}$ от длины волны, чтобы сделать стабильными параметры сенсора в широком спектральном интервале. Тогда матрица принимает простой вид

$$
Z_{L H, N}=\left[\begin{array}{cc}
\left(-\frac{u_{H}}{u_{L}}\right)^{N} & 0 \\
0 & \left(-\frac{u_{L}}{u_{H}}\right)^{N}
\end{array}\right]
$$

$u_{j}, j=L, H-$ определяются по аналогии с (5). Полные матрицы $Z_{1}^{[S, P]}$ системы с металлической пленкой для двух поляризаций

$$
Z_{1}^{[S, P]}=Z_{m}^{[S, P]} Z_{L H, N}^{[S, P]}
$$

Коэффициенты отражения $R_{1}^{[S, P]}$ определяются подстановкой элементов матрицы $Z_{1}^{[S, P]}$ в (6) и возведением всего выражения по модулю в квадрат, при этом $u_{a} \rightarrow u_{b}$, так как в данном случае база является средой, в которую свет выходит.
Подобные выражения можно получить и для определения $R_{2}^{[S, P]}$ зеркала $M_{1}$ на основе матриц $Z_{H L, N}^{[S, P]}$, полученных из (9) взаимной заменой индексов $L$ и $H$, для света, падающего со стороны базы интерферометра

$$
Z_{2}^{[S, P]}=Z_{H L, N}^{[S, P]} Z_{m}^{[S, P]} .
$$

Коэффициенты отражения $R_{2}^{[S, P]}$ определяются подстановкой $Z_{2}^{[S, P]}$ в (6) и возведением по модулю в квадрат, при этом необходимо взаимно поменять $u_{p r}$ и $u_{a}$ $\left(u_{a} \rightarrow u_{b}\right)$.

В результате получаются следующие выражения для энергетических коэффициентов и их фаз на центральной длине волны $\lambda_{0}$, вблизи которой будет максимум спектральных профилей $R_{1,2}^{[S, P]}$ :

$$
\begin{aligned}
& R_{1}^{[S, P]}=\frac{\left(u_{p r}-\xi_{1}-u_{b}\left(u_{L} / u_{H}\right)^{2 N}\right)^{2}+\xi_{2}^{2}}{\left(u_{p r}+\xi_{1}+u_{b}\left(u_{L} / u_{H}\right)^{2 N}\right)^{2}+\xi_{2}^{2}}, \\
& \operatorname{tg} \Psi_{1}^{[S, P]}=\frac{2 u_{p r} \xi_{2}}{\left(\xi_{1}+\left(u_{L} / u_{H}\right)^{2 N} u_{b}\right)^{2}-u_{p r}^{2}+\xi_{2}^{2}}, \\
& R_{2}^{[S, P]}=\frac{\left(u_{p r}+\xi_{1}-u_{b}\left(u_{L} / u_{H}\right)^{2 N}\right)^{2}+\xi_{2}^{2}}{\left(u_{p r}+\xi_{1}+u_{b}\left(u_{L} / u_{H}\right)^{2 N}\right)^{2}+\xi_{2}^{2}}, \\
& R_{2}^{[S, P]} \approx 1-\frac{4\left(u_{p r}+\xi_{1}\right) u_{b}}{\left(u_{p r}+\xi_{1}\right)^{2}+\xi_{2}^{2}}\left(\frac{u_{L}}{u_{H}}\right)^{2 N}+O\left(\left(\frac{u_{L}}{u_{H}}\right)^{4 N}\right) \text {, } \\
& u_{L}<u_{H} \text {, } \\
& \operatorname{tg} \Psi_{2}^{[S, P]}=\frac{2\left(u_{L} / u_{H}\right)^{2 N} u_{b} \xi_{2}}{\left(u_{p r}+\xi_{1}\right)^{2}-\left(u_{L} / u_{H}\right)^{4 N} u_{b}^{2}+\xi_{2}^{2}}, \\
& T_{1}^{[S, P]}=T_{2}^{[S, P]}=\frac{4\left(u_{L} / u_{H}\right)^{2 N} u_{p r} u_{b}}{\left(u_{p r}+\xi_{1}+u_{b}\left(u_{L} / u_{H}\right)^{2 N}\right)^{2}+\xi_{2}^{2}}, \\
& \operatorname{tg} \Phi_{1}^{[S, P]}=-\frac{\xi_{2}}{\left(u_{p r}+\xi_{1}+\left(u_{L} / u_{H}\right)^{2 N} u_{b}\right.}, \\
& A_{1}^{[S, P]}=1-R_{1}^{[S, P]}-T_{1}^{[S, P]}=\frac{1+R_{2}^{[S, P]} u_{p r} / \xi_{1}}{1+u_{p r} / \xi_{1}}-R_{1}^{[S, P]} \text {, } \\
& A_{2}^{[S, P]}=1-R_{2}^{[S, P]}-T_{2}^{[S, P]}=\frac{1-R_{2}^{[S, P]}}{1+u_{p r} / \xi_{1}},
\end{aligned}
$$

где $u_{L, H, p r}$ и $\xi_{1,2}$ должны подставляться для соответствующих поляризаций. Формулы (12)-(15) являются обобщением аналогичных формул для нормального падения, приведенных в [9].

Из (11) и (12) следует, что в условиях согласования при $\xi_{1}=u_{p r}$ и $\xi_{2} \rightarrow 0\left(u_{H}>u_{L}\right)$ значение $R_{1}^{[S, P]} \rightarrow 0$, а фаза $\Psi_{1}^{[S, P]} \rightarrow \pi / 2+m \pi, m-$ целое число. С другой стороны, при любых допустимых $\xi_{1,2} R_{2}^{[S, P]} \rightarrow 1$ при росте $N$, и фаза $\Psi_{2}^{[S, P]} \rightarrow \pi+m \pi$, т. е. стремится к значениям обычного зеркала без поглощения. Если учесть еще и пропускание $T_{1}^{[S, P]}(14)$, то можно получить поглощение в структуре со стороны многослойного покрытия для 

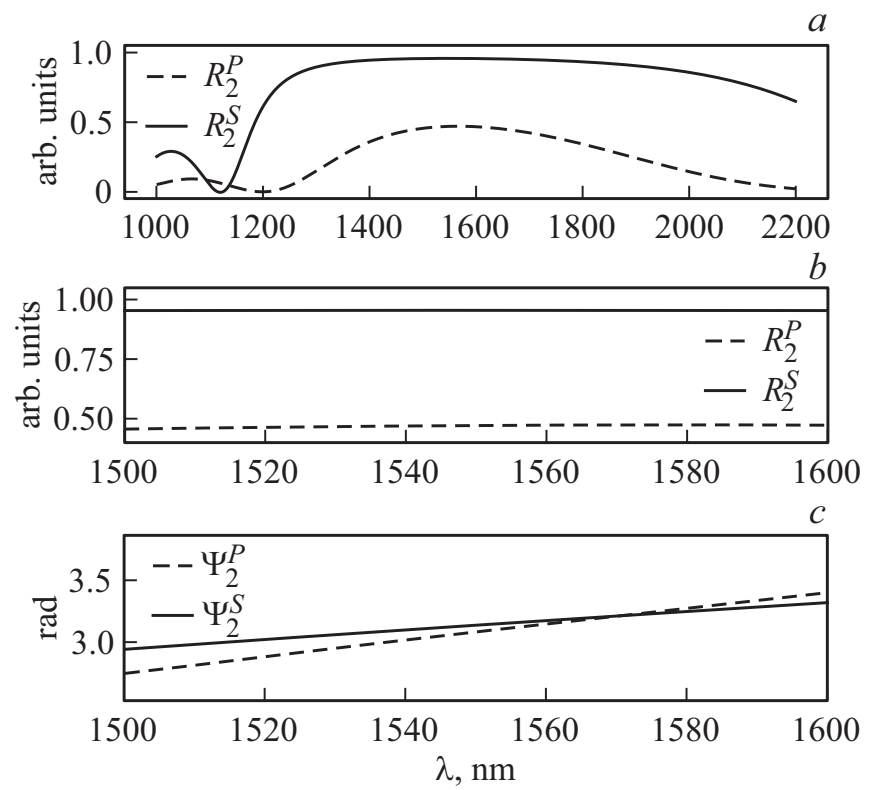

Pис. 3. Расчетные спектры коэффициентов отражения $R_{2}^{[S, P]}$ для $N=3$ и двух поляризаций в спектральном диапазоне: (a) $1000-2200 \mathrm{~nm},\left(\right.$ b) $1500-1600 \mathrm{~nm}$ и $(c)$ фаз $\Psi_{2}^{[S, P]}(\lambda)$.

бегущей волны $A_{2}^{[S, P]}$. Как видно из $(15)$, при согласовании потери равны половине от неотраженной энергии и уменьшаются с ростом $R_{2}^{[S, P]}$. С математической точки зрения тонкая металлическая пленка создает асимметрию коэффициента отражения за счет появления действительной части у левого нижнего элемента матрицы $Z_{m}^{[S, P]}$, обусловленного наличием поглощения $\chi_{m}>0$ у пленки.

Параметр $\vartheta^{[S, P]}$ влияет на асимметричность профиля максимума, если подставить выражения для фаз отражения и пропускания и упростить выражение, то получается

$$
\operatorname{tg} \vartheta^{[S, P]}=-\frac{2 \xi_{1} \xi_{2}}{\left(u_{p r}-\left(u_{L} / u_{H}\right)^{2 N} u_{b}\right)^{2}-\xi_{1}^{2}+\xi_{2}^{2}}
$$

Равенство согласования $\xi_{1}=u_{p r}$ можно выполнить как для $S$-, так и для $P$-поляризаций путем подбора толщины пленки. Неравенство $\xi_{2} \ll 1$ можно выполнить, если использовать металлы с близкими $n_{m} \approx \chi_{m}$. Для этого хорошо подходит титан в видимой и ближней ИК областях $\hat{n}_{T i}=3.68-4.61 i \quad 1550 \mathrm{~nm}$ [13].

\section{4. Основные характеристики сенсора}

Для определения основных характеристик сенсора необходима область свободной дисперсии интерферометра, она приближенно может быть получена из выражения

$$
\Delta \lambda \approx \frac{\lambda^{2}}{2 n_{b} h_{b} \cos \theta_{b}}[n m]
$$

где $\lambda$ - длина волны максимума. Спектральные чувствительности данного сенсора $S_{\lambda}^{[S, P]}$ могут быть выражены аналогично [5], рассматривая изменения фазы отражения зеркала $M_{2}$ только за счет ПВО:

$$
\begin{aligned}
S_{\lambda}^{[S]} \approx-\frac{\Delta \lambda}{2 \pi} \frac{\partial \Psi_{3}^{[S]}}{2 n_{a}} & =\frac{\Delta \lambda}{\pi} \frac{n_{a} \sqrt{f_{b}}}{n_{b}^{2}-n_{a}^{2}} \frac{1}{\sqrt{f_{a}}}\left[\frac{\mathrm{nm}}{R I U}\right] \\
S_{\lambda}^{[P]} \approx-\frac{\Delta \lambda}{2 \pi} \frac{\partial \Psi_{3}^{[P]}}{\partial n_{a}} & =\frac{\Delta \lambda}{\pi} \frac{n_{b}^{2} n_{a} \sqrt{f_{b}}}{\left(n_{a}^{4} f_{b}+n_{b}^{4} f_{a}\right)} \frac{1}{\sqrt{f_{a}}}\left[\frac{\mathrm{nm}}{R I U}\right] \\
f_{a} & =\left(n_{p r} \sin \theta_{p r}\right)^{2}-n_{a}^{2} \\
f_{b} & =n_{b}^{2}-\left(n_{p r} \sin \theta_{p r}\right)^{2}
\end{aligned}
$$

$R I U$ - refractive index units, где последняя дробь в произведениях теоретически может расти до бесконечности при приближении $\theta_{p r}$ к углу $\theta_{\text {Пво }}=\arcsin \left(n_{a} / n_{p r}\right)$. Однако при этом уменьшается диапазон изменения $n_{a}$ в сторону увеличения (приближения к $n_{p r}$ ).

Спектральная ширина $\delta \lambda$ максимумов интенсивности связана с $\Delta \lambda$ соотношением

$$
\delta \lambda^{[S, P]}=\frac{\Delta \lambda}{F^{[S, P]}}[\mathrm{nm}]
$$

где $F^{[S, P]}$ - резкость полос, определяющая эффективное количество интерферирующих лучей:

$$
\begin{gathered}
F^{[S, P]}=\pi \frac{\left(R_{2}^{[S, P]} R_{3}^{[S, P]}\right)^{1 / 4}}{1-\left(R_{2}^{[S, P]} R_{3}^{[S, P]}\right)^{1 / 2}}, \\
F^{[S, P]} \approx \pi \frac{\left(u_{p r}+\xi_{1}\right)^{2}+\xi_{2}^{2}}{2\left(u_{p r}+\xi_{1}\right) u_{b}}\left(\frac{u_{H}}{u_{L}}\right)^{2 N}, \quad u_{L}<u_{H} .
\end{gathered}
$$

Она растет с увеличением $N$ и ограничивается только потерями в структуре интерферометра.

Контраст $C_{R}^{[S, P]}$ спектральной характеристики напрямую влияет на $F O M(1)$, из теории ОИ [9]:

$$
\begin{gathered}
C_{R}^{[S, P]}=\frac{R_{\max }-R_{\min }}{R_{\max }}, \\
R_{\max }=\left(g_{1}+g_{2}\right)^{2}, \quad R_{\min }=\left(g_{1}-g_{2}\right)^{2}, \\
g_{1}=\sqrt{R_{1}+\frac{2 T_{1} \sqrt{R_{1} R_{2}} R_{3}}{1-R_{2} R_{3}} \cos \vartheta+\frac{T_{1}^{2} R_{2} R_{3}^{2}}{\left(1-R_{2} R_{3}\right)^{2}}}, \\
g_{2}=\frac{T_{1} \sqrt{R_{3}}}{1-R_{2} R_{3}},
\end{gathered}
$$

где $R_{\max , \min }-$ максимальное и минимальное значения $R(\lambda)$, а $R_{1,2,3}, T_{1}$ берутся для соответствующих поляризаций. Величина $R_{\min }$ зачастую может быть очень мала в пределах спектрально широкой области свободной дисперсии в сотни нанометров. Но в измерениях спектрального положения максимума используется только малая спектральная окрестность около него. Поэтому вместо $R_{\min }$ следует использовать $R_{1}$, как определяющую 

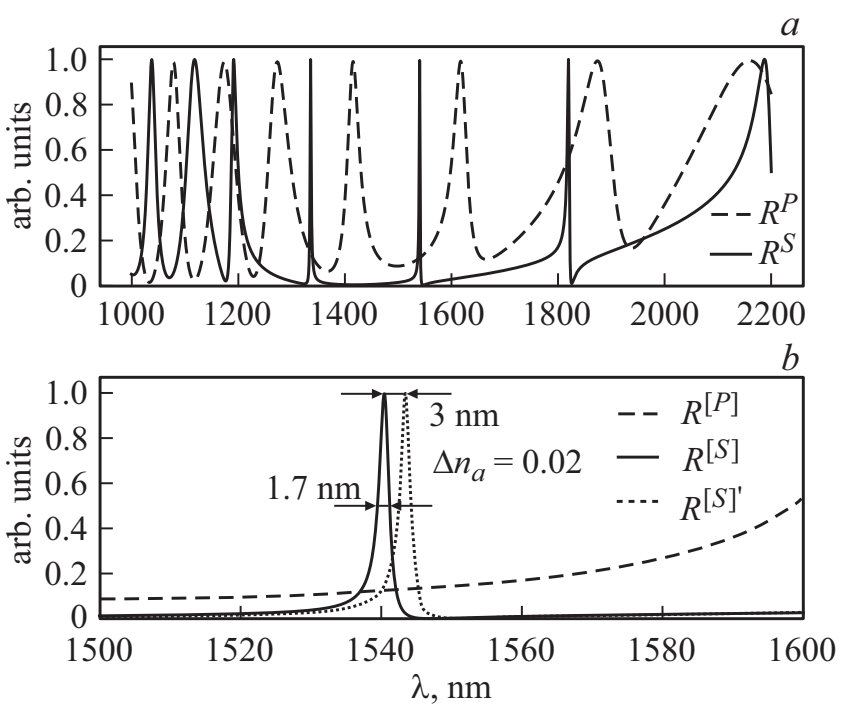

Рис. 4. Расчетный спектр коэффициентов отражения интерферометра $R$ для $S$ - и $P$ - поляризаций в спектральном диапазоне: (a) $1000-2200 \mathrm{~nm}$, (b) $1500-1600 \mathrm{~nm}$. Пунктиром показан спектрально смещенный пик при изменении $n_{a}$ на $0.02 R I U$.

основной вклад в формирование фоновой подкладки вблизи максимума.

По формулам (1), (12), (16)-(21) можно с хорошей точностью определить основные параметры сенсора на центральной длине волны $\lambda_{0}$. Однако более точный учет фаз отражения и пропускания зеркал $M_{1,2}$ сдвигает пик резонанса по спектру от центральной длины волны, поэтому важно продемонстрировать спектральные распределения параметров, которые получаются из численных расчетов в одном из частных случаев.

\section{5. Численное моделирование характеристик сенсора}

Для примера моделирование спектрального распределения было выполнено в приближении массивного слоя, т.е. без приближения МПП (7), чтобы результат был как можно более точным. Толщина титановой пленки была подобрана, основываясь на аналитических формулах теории МПП, чтобы выполнялось условие согласования для $S$-поляризации $\xi_{1}^{[S]}=n_{p r} \cos \theta_{p}=1.1$ $\left(n_{p r}=1.52, \xi_{2}^{[S]}=-0.25\right)$, для угла падения в призме $\theta_{p r}=43^{\circ}$, близком к углу ПВО $\left(\theta_{t i r}=41.14^{\circ}, n_{a}=1\right)$ (рис. 2 и 3). Структура слоев: [призма, Тi, $L, H, L, H, L$, $H$, аналит] соответствуют $N=3$ в формулах (12)-(14), где $T_{i}$ - пленка титана, $h_{\mathrm{Ti}}=8 \mathrm{~nm}, h_{L}=\lambda_{0} /\left(4 n_{L} \cos \theta_{L}\right)$, $h_{H}=\lambda_{0} /\left(4 n_{H} \cos \theta_{H}\right), n_{L}=1.46\left(\mathrm{SiO}_{2}\right), n_{H}=2.4\left(\mathrm{TiO}_{2}\right)$, $n_{b}=n_{H}, \lambda_{0}=1550 \mathrm{~nm}$. При расчете значения коэффициентов преломления не изменялись во всем спектральном интервале. Из рис. 2, a следует, что $R_{1}^{[S, P]}$ для двух поляризаций достигает минимума в области вблизи центральной длины волны, а в области 1500-1600 nm - менее $0.035: R_{1}^{[S]}=0.017(0.0171)$ и $R_{2}^{[P]}=0.016(0.024)$ $1550 \mathrm{~nm}$ (рис. 2,b), где в скобках приведены значения по формулам (12), (13). Рис. 3, a демонстрирует, что максимумы $R_{2}^{[S, P]}$ находятся на центральной длине волны, спектральная область высокого коэффициента отражения более $1000 \mathrm{~nm}$, что обусловлено как разницей коэффициентов преломления диэлектрических слоев, так и косинусами углов преломления. Значительная разница как в максимальных значениях $R_{2}^{[S]}=0.957 \quad(0.954)$ и $R_{2}^{[P]}=0.472 \quad(0.454) \quad 1550 \mathrm{~nm}$ (рис. $3, b)$, так и в спектральной ширине на полувысоте объясняется разными величинами отношений $u_{H}^{[S]} / u_{L}^{[S]}$ и $u_{H}^{[P]} / u_{L}^{[P]}$, как следует из формулы (13). Для S-поляризации $u_{H}^{[S]} / u_{L}^{[S]}=n_{H} \cos \theta_{H} /\left(n_{L} \cos \theta_{L}\right)$, для $P-$ $u_{H}^{[P]} / u_{L}^{[P]}=n_{H} \cos \theta_{L} /\left(n_{L} \cos \theta_{H}\right)$. Так как отношение этих двух выражений больше единицы:

$$
\frac{u_{H}^{[S]} / u_{L}^{[S]}}{u_{H}^{[P]} / u_{L}^{[P]}}=\left(\frac{\cos \theta_{H}}{\cos \theta_{L}}\right)^{2}>1,
$$

где $\cos \theta_{H}>\cos \theta_{L}$, то всегда $R_{2}^{[S]}>R_{2}^{[P]}$, т. е. $R_{2}^{[S]}$ гораздо быстрее стремится к 1 с ростом $N$, чем $R_{2}^{[P]}$, а спектральная ширина $R_{2}^{[S]}$ больше, чем $R_{2}^{[P]}$. Это значит, что благодаря большей резкости полос $(20)$, обусловленной более высокой добротностью, в отражении интерферометра для $S$-поляризации будут наблюдаться более узкие максимумы отражения при том же числе слоев $N$ диэлектриков вблизи длины волны $\lambda_{0}$, чем для $P$ поляризации. Поэтому выгоднее использовать сенсор на основе $S$-поляризации, как имеющий больший $F O M$. Тем не менее потенциально возможно использовать и $P$ поляризацию, если того будет требовать задача, но для оптимизации контраста, т.е. зануления $R_{1}^{[P]}$, надо выполнить согласование $\xi_{1}^{[P]}=n_{p r} / \cos \theta_{p r}$. Как следует из формул (17) и (18), чувствительность $S_{\lambda}^{[P]}$ при большом $n_{b}$ может быть в разы больше $S_{\lambda}^{[S]}$, что потенциально может сделать более выгодной $P$-поляризацию с точки зрения $F O M$.

Так как по формуле (2) положение максимума сенсора зависит от набега фаз между зеркалами, то важна и спектральная зависимость фаз зеркал. На рис. 3, $c$ приводятся фазы отражения $\Psi_{2}^{[S, P]}(\lambda)$ для двух поляризаций в диапазоне центральной длины волны 1500-1600 nm. Изменение фаз на всем спектральном интервале мало изменяется с ростом $\mathrm{N}$ и составляет $<0.4 \mathrm{rad}$, хорошо аппроксимируется полиномиальной зависимостью, т.е. их влияние на спектральную чувствительность сенсора может быть исключено при калибровке. Спектральную зависимость имеют также и другие фазы переднего зеркала. Они вносят свой вклад в положение максимума (1) через фазу Ами $\vartheta(\lambda)$ во втором слагаемом, так как асимметричность может немного сдвигать положение максимума, но этим влиянием можно пренебречь при $R_{1} \ll 1$. 
Ширина максимума и параметр качества для $S$ - и $P$ поляризаций при различных количествах слоев сенсора

\begin{tabular}{c|c|c|c|c}
\hline \multirow{2}{*}{$N$} & \multicolumn{2}{|c|}{$\delta \lambda, \mathrm{nm}$} & \multicolumn{2}{c}{ FOM, $\mathrm{RIU}^{-1}$} \\
\cline { 2 - 5 } & $\mathrm{S}$ & $\mathrm{P}$ & $\mathrm{S}$ & $\mathrm{P}$ \\
\hline 1 & 437 & 1141 & 3.6 & 5.2 \\
\hline 2 & 94.5 & 633 & 16.2 & 9.4 \\
\hline 3 & 21.7 & 373 & 72 & 16 \\
\hline 4 & 4.9 & 224 & 318.2 & 26.7 \\
\hline 5 & 1.1 & 135.5 & 1410 & 44 \\
\hline 6 & 0.25 & 82.1 & 6251.7 & 72.8 \\
\hline 7 & 0.056 & 49.8 & 27712 & 120
\end{tabular}

На рис. 4 показана спектральная характеристика отражения сенсора с базой в $11 h_{H}\left(n_{b}=2.4\right)$, соответствующей примерно $\Delta \lambda=280(282) \mathrm{nm}$ области свободной дисперсии. При изменении коэффициента преломления $n_{a}$ на $0.02 R I U$ сдвиг составляет около $3 \mathrm{~nm}$, что соответствует $\delta \lambda^{[S]}=1.7 \quad(1.95) \mathrm{nm}$, чувствительности $S_{\lambda}^{[S]} \approx 150(149.3)\left[\frac{\mathrm{nm}}{R I U}\right]$ и $F O M^{[S]} \approx 90(72)\left[R I U^{-1}\right]$, где в скобках приведены значения, полученные по формулам (16), (17), (19), (20). Чувствительность может быть увеличена до $S_{\lambda}^{[S]}=1643\left[\frac{\mathrm{nm}}{R I U}\right]\left(S_{\lambda}^{[P]}=6289\left[\frac{\mathrm{nm}}{R I U}\right]\right.$, если уменьшить базу до одного $h_{H}$, и это будет предельная чувствительность за счет уменьшения базы для данного угла $(\Delta \lambda \approx 3100 \mathrm{~nm})$. Для этого случая в таблице приводятся сравнительные характеристики ширин максимумов и параметров качества для двух поляризаций от числа слоев $N$ (вычисления сделаны для коэффициентов отражений $R_{2}^{[S, P]}$ на центральной длине волны).

\section{6. Метод изготовления}

Для изготовления сенсора предполагается, что будет задан спектральный диапазон работы, центральная длина волны $\lambda_{0}$, коэффициент преломления призмы $n_{p r}$ и аналита $n_{a}$. Угол падения в призме $\theta_{p r}$ выбирается исходя из требуемого диапазона изменения коэффициента преломления, а также чувствительности сенсора. Количество слоев диэлектрической структуры выбирается из расчета получить заданную спектральную ширину $\delta \lambda$ сигнального максимума. Затем можно определить условия изготовления согласованной металлической пленки для выбранного угла падения и нанести ее на грань целевой призмы или на целевую плоскопараллельную подложку, в последнем случае ее далее можно совместить с призмой посредством оптического контакта. Диэлектрическую часть структуры можно изготовить вакуумным напылением при оптическом контроле за процессом нанесения слоев по плоскопараллельной подложке-свидетелю из того же материала, что и целевая подложка, с помощью лазерного излучения на центральную длину волны работы сенсора. Диэлектрическое покрытие, содержащее слои зеркала $M_{1}$ и базы, должно наноситься, ориентируясь по экстремумам пропускания или отражения, при этом угол падения на структуру внутри подложки-свидетеля должен быть равен требуемому углу падения в призме $\theta_{p r}$, если коэффициенты преломления подложки и призмы совпадают, или пересчитываться по закону преломления в случае разных материалов, что автоматически сформирует слои с нужной толщиной.

\section{7. Заключение}

В работе рассмотрены аналитические формулы для описания свойств сенсора показателя преломления в схеме Кречмана на основе отражательного интерферометра для наклонного падения света. Структура слоев интерферометра представляет собой тонкую металлическую пленку и четвертьволновое покрытие для наклонного падения света. Показано, что толщина и коэффициент преломления металлической пленки должны быть выбраны определенным образом, чтобы оптимизировать параметры сенсора с точки зрения максимизации контраста сигнального максимума, при этом параметры пленки зависят от поляризации. Эффективное количество интерферирующих лучей в интерферометре также зависит от поляризации, и выгоднее с точки зрения параметра качества для того же количества слоев диэлектрической структуры использовать S-поляризацию. Потенциально можно получать сравнимые с $S$-поляризацией параметры качества и для $P$-поляризация, однако потребуется большее количество слоев диэлектрической структуры. Предложен один из вариантов метода изготовления данного сенсора с оптическим контролем процесса на целевых для работы сенсора длине волны и угле падения света.

\section{Финансирование работы}

Работа выполнена в рамках темы госзадания Института автоматики и электрометрии СО РАН (базовый бюджет, „Физические основы лазерных и сенсорных систем с использованием структурированных волоконных световодов и микрорезонаторов“) (№ 121030500067-5).

\section{Конфликт интересов}

Авторы заявляют, что у них нет конфликта интересов.

\section{Список литературы}

[1] Homola J. Surface Plasmon Resonance Based Sensors, Springer, 2006.

[2] Prabowo B.A., Purwidyantri A., Liu K.-C. // Biosensors. 2018. V. 8. N 3. P. 80 . doi $10.3390 /$ bios 8030080 
[3] Hlubina P., Urbancova P., Pudis D., Goraus M., Jandura D., Ciprian D. // Opt. Lett. 2019. V. 44. N 22. P. 5602. doi 10.1364/OL.44.005602

[4] Rahimi L., Askari A.A. // Appl. Opt. 2020. V. 59. N 34. P. 10980. doi 10.1364/AO.405129

[5] Терентьев В.С., Симонов В.А. // ОПт. и спектр. 2021. T. 129. № 2. C. 238. doi 10.21883/OS.2021.02.50564.232-20

[6] Printz M., Sambles J.R. // J. Mod. Opt. 1993. V. 40. N 11. P. 2095. doi 10.1080/09500349314552131

[7] Голдина Н.Д. // Автометрия. 2009. Т. 45. № 6. С. 99; Goldina N.D. // Optoelectron. Instrument. Proc. 2009. V. 5. P. 71. doi 10.3103/S8756699009060120

[8] Голдина Н.Д. // Автометрия. 2021. № 2. С. 122.

[9] Троицкий Ю.В. Многолучевые интерферометры отраженного света. Новосибирск: Наука, 1985. 208 с.

[10] Holden J. // The Proceedings of the Physical Society. Section B. 1949. V. 62. Part 7. № 355B. P. 405.

[11] Борн М., Вольф Э. Основы оптики. М.: Наука, 1973. 720 с.

[12] Розенберг Г.В. Оптика тонкослойных покрытий. М.: Гос. изд-во физ.-мат. лит., $1958.570 \mathrm{c}$.

[13] Johnson P.B., Christy R.W. // Phys. Rev. B. 1974. V. 9. P. 5056. doi 10.1103/PhysRevB.9.5056 